04

\title{
Ускорение ионных колец сжимающимися лайнерами
}

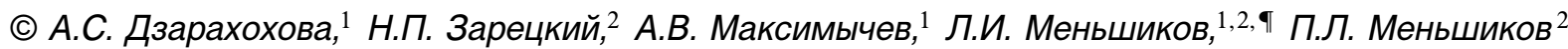 \\ ${ }^{1}$ Московский фризико-технический институт (государственный университет), \\ 141700 Долгопрудный, Московская обл., Россия \\ 2 Национальный исследовательский центр „Курчатовский институт“, \\ 123182 Москва, Россия \\ ๑ e-mail: mleonid1954@mail.ru
}

Поступило в Редакцию 25 января 2019 г.

В окончательной редакции 21 ноября 2019 г.

Принята к публикации 3 декабря 2019 г.

Установлены условия отсутствия альвеновской ионно-циклотронной неустойчивости при создании и последующем ускорении ионных колец сжимающимися цилиндрическими плазменными лайнерами.

Ключевые слова: ускоритель ионов, дисперсионное уравнение, плазменные лайнеры, ионные кольца, импульсные рентгеновские источники, альвеновская ионно-циклотронная неустойчивость плазмы, конфигурации с обращенным магнитным полем.

DOI: $10.21883 / J T F .2020 .06 .49275 .25-19$

\section{1. Постановка задачи}

Основным мотивом написания настоящей работы явилась детализация схемы малоразмерного (по сравнению, например, с сильноточными протонными ускорителями) мощного импульсного ускорителя ионов, предложенного в работах [1,2] (рис. 1). Нейтрализованный по заряду электронами пучок ионов, выходящий в виде полого цилиндра из аксиально-симметричного импульсного ускорителя ионов прямого действия (диода), движется вдоль силовых линий магнитного поля левого соленоида. В промежутке между соленоидами, магнитные поля которых направлены противоположно друг другу, имеется радиальное магнитное поле, которое превращает пучок в кольцо. При наличии слабого градиента поля в правом соленоиде это кольцо замедляется и останавливается в „магнитной пробке“ на характерное время $~ 10-50 \mathrm{~ns}$, в течение которого происходит сжатие и ускорение ионного кольца вихревым электрическим полем, возникающим при сжатии магнитного потока, захваченного высокопроводящим плазменным лайнером. В $[1,2]$ в качестве лайнера предложено использовать газовый, позволяющий осуществить высокую частоту повторения импульсов ускорения.

Образование ионных колец по схеме, представленной на рис. 1, доказано в опытах с протонами [3,4]. Возможность сжатия магнитного потока проверена, например, в эксперименте [5]. Через струю газообразного неона в виде полого цилиндра длиной $L=2 \mathrm{~cm}$ с начальным радиусом $R_{i}=2 \mathrm{~cm}$ пропускался созданный генератором сверхвысокой электрической мощности (СВЭМ) импульсный разряд с током 7.5 МА. Было получено конечное (в момент наибольшего сжатия) магнитное поле $B_{f}=40 \mathrm{MG}$ при начальной (до сжатия) величине $B_{i}=100 \mathrm{kG}$. Таким образом, при двадцатикратной степени радиального сжатия было достигнуто усиление магнитного поля в $20^{2}=400$ раз, что доказывает на- дежность удержания магнитного потока сжимающимся лайнером. Установлено, что наличие сжимаемого оболочкой магнитного поля обеспечивает повышенную устойчивость сжатия по сравнению со сжатием без захваченного лайнером магнитного поля.

В качестве возможных приложений рассматриваемого ускорителя можно указать:

1. Создание на основе ускорителя протонов импульсного источника нейтронов, мощность которого существенно превышает таковую для известных схем, например, плазменного фокуса (см., например, обзор [6] и ссылки в нем). Этот источник можно было бы применять для подпитки подкритических реакторов [7] (для понижения амплитуды скачков мощности в таком случае предпочтительны гомогенные активные зоны, например, жидкосолевые), для создания мощного импульсного источника антинейтрино по схеме, предложенной в [8], и позже развитой и детализированной в [9], а также в других целях;

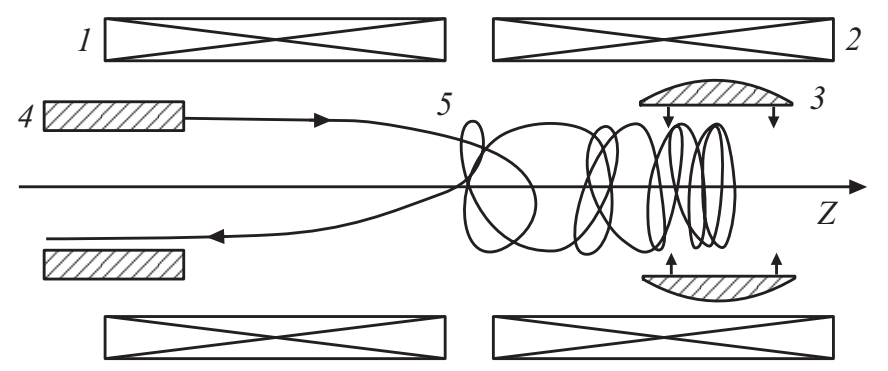

Рис. 1. Принципиальная схема импульсного ускорителя ионов на основе сжимающегося газового лайнера 3 в виде полого цилиндра. Соленоиды 1 и 2 с полем $B_{i} \sim 10 \mathrm{~T}$, включенные навстречу друг другу, создают магнитное поле с конфигурацией типа „касп“. Кривая 5 - траектория одного из ионов, испускаемых импульсным коаксиальным диодом 4 с магнитной изоляцией. 
2. Создание импульсного рентгеновского источника сверхвысокой мощности.

В настоящей работе для конкретности рассматривается последнее направление - рентгеновский источник. Количественные оценки будут приводиться для проекта, представленного в работе [10], где на базе схемы $[1,2]$ предложено создать и ускорить кольцо ионов $\mathrm{Xe}^{+Z}$ с зарядом $Z=8$ (из многообразия обсуждаемых в [10] ионов для определенности будем рассматривать эти ионы; они получаются при полной обдирке внешней оболочки у атомов ксенона) и ускорить их сжимающимся газовым лайнером до энергии $\sim 1 \mathrm{GeV}$. Ионы выходят из коаксиального диода со скоростью $u \sim 10^{8} \mathrm{~cm} / \mathrm{s}$. Начальные (перед ускорением) параметры плазмы в кольце таковы: концентрации ионов и электронов равны соответственно $n_{i}^{0} \sim 2 \cdot 10^{14} \mathrm{~cm}^{-3}$ и $n_{e}^{0} \sim 1.5 \cdot 10^{15} \mathrm{~cm}^{-3}$, масса ионов $M=2.3 \cdot 10^{-22} \mathrm{~g}, B_{i} \sim 5 \cdot 10^{4} \mathrm{G}$. На оси симметрии $\mathrm{z}$ лайнера длиной $L=4 \mathrm{~cm}$ располагается мишень из материала с атомами В с большим атомным номером. Сжатое и ускоренное магнитным полем кольцо ионов входит в эту мишень. В столкновениях $\mathrm{Xe}^{+Z}+B$ на $K$ оболочках этих ионов и атомов образуются вакансии, в результате чего возникает характеристическое рентгеновское излучение с квантами в единицы и десятки килоэлектрон-вольт (используя различные ионы и атомы мишени, энергию квантов можно менять в широких пределах). При таком способе энергия рентгеновских лучей составляет $\sim 20 \%$ от кинетической энергии, переданной лайнеру от СВЭМ, что более чем на порядок превышает КПД наилучших схем с тепловым спектром квантов (см., например, [11]). Суммарная энергия излучения на существующих установках может достигать $\gtrsim 10 \mathrm{MJ}$. Любопытным свойством схемы [10] является то, что излучение испускается в виде узкого луча с углом раствора $\sim 2^{\circ}$ в течение нескольких насносекунд. Таким образом, при достижении величины частоты повторения импульсов порядка один раз в минуту средняя яркость источника составит $10^{25}$ фотонов $/\left(\mathrm{s} \cdot \mathrm{mm}^{2} \cdot \mathrm{mrad}^{2}\right)$, т.е. столько же как, скажем, намечается в проекте Европейского рентгеновского лазера на свободных электронах - XFEL. Поскольку линии характеристического спектра узкие, $\Delta \lambda / \lambda \sim 10^{-3}$, то, как и у XFEL, излучение характеризуется большой длиной когерентности $\lambda^{2} / \Delta \lambda=10^{3} \lambda$, что позволяет, например, исследовать структуру белков. Указанный источник мог бы найти применение также в исследованиях по инерциальному управляемому термоядерному синтезу (ICF), в рентгеновской литографии и в других областях.

При образовании и последующем сжатии кольца ионы взаимодействуют как с электронами плазмы, так и при посредстве электронов между собой. Условия отсутствия неустойчивостей, вызванных взаимодействием с электронами, выяснены в работе [12]. Там же показано, что в условиях проекта $[10]$ неустойчивости этого типа не возникают. Цель настоящей работы состоит в установлении условий подавления коллективных неустойчивостей, обусловленных опосредствованными электронами ион-ионными взаимодействиями.

\section{2. Вывод дисперсионного уравнения для бесконечно длинного в направлении магнитного поля ионного кольца}

Значение эффективного параметра бета (отношение давлений плазмы и магнитного поля), характерного для плазмы в ионном кольце проекта [10], достаточно велико

$$
\beta=\frac{4 \pi n_{i}\left(M u^{2} / 2\right)}{B^{2}} \sim 1,
$$

где $u$ - скорость ионов, поэтому существен диамагнетизм. В этом случае по мере накопления ионов магнитное поле внутри кольца становится существенно меньше, чем снаружи. Изменение магнитного поля во времени приводит к возникновению азимутального вихревого электрического поля, которое тормозит часть ионов. Соотношение (1) свидетельствует о возможном образовании ионного кольца с обращенным полем. В нашем случае характерное время ввода пучка порядка периода обращения ионов в магнитном поле. По этой причине при вводе пучка нарушается аксиальная симметрия, что является необходимым условием для образования конфигурации с обращенным полем $[13,14]$. И, действительно, такие кольца образовывались в опытах $[3,4]$. В более поздних исследованиях, представленных в обзорах $[15,16]$, этот процесс был изучен более детально. Траектории ионов при этом становятся довольно сложными [17-19], а распределения ионов и электронов по скоростям значительно уширяются (при испускании ионов из диода также создается начальный значительный разброс по скоростям), поэтому характерные значения параметров для плазмы в кольце таковы:

$$
\delta_{i} \sim \delta_{e} \sim 1
$$

Здесь $\delta_{i}=\Delta_{i} / u, \delta_{e}=\Delta_{e} / u, \Delta_{i}$ и $\Delta_{e}-$ характерные величины разбросов скоростей ионов и электронов по скоростям (среднеквадратичные отклонения от средних значений). Учтено, что вследствие электронейтральности плазмы скорость электронов близка к таковой для ионов, $u_{e} \sim u$. Добавим, что, согласно [15,20,21], процессы сжатия ионного кольца и ускорения ионов остаются устойчивыми даже при обращении направления поля внутри кольца.

Согласно имеющимся результатам теории и экспериментов [22-24] (см. также обзор [25,26]), наиболее опасной неустойчивостью кольца, вызванной взаимодействиями между ионами, является альвеновская ионноциклотронная неустойчивость (АИЦН), представляющая собой одну из разновидностей электромагнитных (непотенциальных) неустойчивостей плазмы в магнитном поле. Она играет основную роль при большой величине параметра $\beta$, т.е. как раз в нашем случае, и возникает когда кинетическая энергия движения ионов поперек магнитного поля превышает таковую для продольного движения. Избыток энергии поперечного 
движения приводит к усилению распространяющейся вдоль магнитного поля $(\mathbf{k} \| \mathbf{B})$ альвеновской ионной циклотронной волны (АИЦ), возникающей случайным образом в результате тепловых флуктуаций, поскольку в этом случае излучение вращающимися ионами АИЦ преобладает над их поглощением [27]. При $\beta \ll 1$ инкремент нарастания АИЦН равен

$$
\gamma=\sqrt{\beta} \omega_{B_{i}}=\frac{u \omega_{i}}{c \sqrt{2}},
$$

где $\omega_{B_{i}}=q B=M c, q=Z e, M, B$ и $c$ - ларморовская частота, заряд и масса ионов, величина магнитного поля и скорость света соответственно. Согласно (3), в большинстве представляющих практический интерес случаях АИЦН развивается быстро по сравнению с временем ион-ионных столкновений.

Как будет показано в нашей другой работе, механизм АИЦН полностью аналогичен таковому для неустойчивости Вайбеля [28], возникающей при анизотропном распределении частиц в отсутствие магнитного поля. Характерным следствием АИЦН неустойчивости является изотропизация распределения ионов по скоростям. По этой причине, например, в амбиполярной ловушке ТМХ в опытах [24] наблюдалась быстрая потеря плазмы.

В однородной плазме магнитное поле однородно при любом $\beta$, а дисперсионное уравнение, определяющее зависимость частоты АИЦ от волнового вектора $k$, имеет вид [22]

$$
\frac{k^{2} c^{2}}{\omega}+F_{0}(\omega)=0
$$

где

$$
\begin{gathered}
F_{0}(\omega)=\frac{\omega_{e}^{2}}{\omega_{B_{e}}}+\frac{\omega_{i}^{2} Q_{0}}{\omega-\omega_{B_{i}}}, \\
Q_{0}=1+\frac{k^{2} u^{2}}{2 \omega\left(\omega-\omega_{B_{i}}\right)} .
\end{gathered}
$$

При $u \rightarrow 0$ выражение (4) описывает движущуюся вдоль направления магнитного АИЦ волну постоянной амплитуды. В частности, при $\omega \ll \omega_{B_{i}}$ получаем известный закон дисперсии для АИЦ волн низкой частоты: $\omega=u_{A} k$, где $u_{A}=B / \sqrt{4 \pi \rho}, \rho=M n_{i}$ - плотность вещества плазмы. С учетом последнего слагаемого в квадратной скобке в уравнении (4) эта волна становится неустойчивой, ее амплитуда экспоненциально растет. Рассчитаем, например, инкремент неустойчивости в предельном случае

$$
\beta \ll 1
$$

После введения безразмерных величин

$$
\tau=\left(\frac{k c}{\omega_{i}}\right)^{2}, \quad \Omega=\frac{\omega}{\omega_{B_{i}}}
$$

уравнение (4) принимает вид

$$
\tau\left[1+\frac{\beta}{(\Omega-1)^{2}}\right]+\frac{\Omega^{2}}{\Omega-1}=0,
$$

где учтено условие квазинейтральности плазмы $n_{e}=Z n_{i}$, согласно которому

$$
\frac{\omega_{e}^{2}}{\omega_{B_{e}}}=\frac{\omega_{i}^{2}}{\omega_{B_{i}}} .
$$

Как говорилось выше, за неустойчивость ответственно последнее слагаемое в квадратной скобке в уравнении (8). В случае (6) это слагаемое становится заметным при $\omega \approx \omega_{B_{i}}$, т.е. $\Omega \approx 1$, поэтому решение ищем в виде $\Omega=1+\Delta$. В результате получаем для неустойчивой ветви колебаний квадратное уравнение

$$
\Omega \approx 1-\frac{1}{2 \tau}+i \sqrt{\beta-\frac{1}{4 \tau^{2}}} .
$$

Наибольший инкремент АИЦН достигается в предельном случае $\tau \rightarrow \infty$, соответсвующем

$$
k \rightarrow \infty
$$

откуда следует, что

$$
\gamma_{\max }=\sqrt{\beta} \omega_{B_{i}}=\frac{u \omega_{i}}{c \sqrt{2}} .
$$

Отметим, что наименьшим имеющим физический смысл пространственным масштабом, характерным для альвеновских волн, является, очевидно, циклотронный радиус ионных орбит $r_{B_{i}}$, поэтому

$$
k \lesssim k_{\max }=1 / r_{B_{i}},
$$

что соответствует

$$
\tau \lesssim \tau_{\max }=\frac{c^{2}}{r_{B_{i}}^{2} \omega_{i}^{2}}=\frac{1}{2 \beta} .
$$

Отсюда и из (10) понятно, что АИЦН отсутствует при

$$
\beta \gtrsim 1
$$

(отметим, что в $[1,2]$ без детального исследования предполагалось противоположное условие). В действительности параметр $\beta$ не может превысить величину порядка единицы, поскольку при достижении значения $\beta \sim 1$ возникает устойчивое при ускорении кольцо с обращенным полем внутри него.

Теперь учтем неоднородность плазмы в ионном кольце и оценим ее влияние на устойчивость.

Сначала обсудим роль неоднородности плазмы в поперечных к магнитному полю направлениям, т. е. будем считать кольцо бесконечно длинным вдоль его оси. Как говорилось в начале разд. 2, при учете диамагнетизма магнитное поле в кольце становится неоднородным с характерным разбросом

$$
\Delta B \sim B
$$

и, кроме того, ионы и электроны становятся немонохроматичными, приобретают разброс по скоростям. В нерелятивистском случае, как у нас, циклотронные частоты 
частиц не зависят от их скоростей, поэтому последним эффектом, т.е. разбросом по скоростям частиц, можно пренебречь. Вследствие (15) характерный разброс $\Delta \omega_{B_{i}}$ по циклотронным частотам ионов также велик (роль разброса по циклотронным частотам электронов выясним позже)

$$
\Delta \omega_{B_{i}} \sim \omega_{B_{i}} .
$$

Аналогично предыдущему разделу естественно ожидать, что разброс по циклотронным частотам приведет к подавлению коллективного эффекта, каковым и является АИЦН.

Слагаемое с $\omega_{i}^{2}$ в (4) пропорционально концентрации ионов $n_{i}$. Концентрация ионов, вращающихся с циклотронной частотой в интервале $\left(\omega_{B_{i}}, \omega_{B_{i}}+d \omega_{B_{i}}\right)$, равна

$$
d n_{i}=n_{i} f\left(\omega_{B_{i}}\right) d \omega_{B_{i}} .
$$

Для функции распрелеления по циклотронным частотам ионов примем выражение

$$
f\left(\omega_{B_{i}}\right)=\frac{\Delta \omega_{B_{i}}}{\pi\left[\left(\omega_{B_{i}}-\omega_{B_{i}}^{0}\right)^{2}+\Delta \omega_{B_{i}}^{2}\right]} .
$$

С учетом этого дисперсионное соотношение (4), предназначенное для оценки влияния поперечной неоднородности плазмы ионного кольца на АИЦН, перепишется в виде

$$
\frac{k^{2} c^{2}}{\omega}+\frac{\omega_{e}^{2}}{\omega_{B_{e}}}+\omega_{i}^{2} \int_{-\infty}^{+\infty} \frac{f\left(\omega_{B_{i}}\right) d \omega_{B_{i}}}{\omega-\omega_{B_{i}}} Q_{o}=0 .
$$

где по принципу причинности сингулярности подинтегрального выражения следует обходить с учетом замены $\omega \rightarrow \omega+i 0$. Пользуясь теорией вычетов, вместо (4) получаем

$$
\frac{q^{2} c^{2}}{\omega^{2}} \approx-\frac{F(\omega)}{\omega}
$$

где

$$
\begin{gathered}
F(\omega)=\frac{\omega_{i}^{2}}{\omega_{B_{i}}^{0}}+\frac{\omega_{i}^{2} Q}{\omega-\omega_{B_{i}}^{0}+i \Delta \omega_{B_{i}}}, \\
Q=1+\frac{k^{2} u^{2}}{2 \omega\left(\omega-\omega_{B_{i}}^{0}+i \Delta \omega_{B_{i}}\right)} .
\end{gathered}
$$

Здесь было учтено соотношение $(9)$, которое теперь принимает вид

$$
\frac{\omega_{e}^{2}}{\omega_{B_{e}}}=\frac{\omega_{i}^{2}}{\omega_{B_{i}}^{0}}
$$

\section{3. Исследование дисперсионного уравнения (18)}

Перепишем уравнение (18) в безразмерных величинах (7)

$$
\tau\left[1+\frac{\beta}{\left(\Omega-1+i \delta_{i}\right)^{2}}\right]+\frac{\Omega\left(\Omega+i \delta_{i}\right)}{\Omega-1+i \delta_{i}}=0,
$$

где

$$
\delta_{i}=\frac{\Delta \omega_{B_{i}}}{\omega_{B_{i}}^{0}} \sim 1
$$

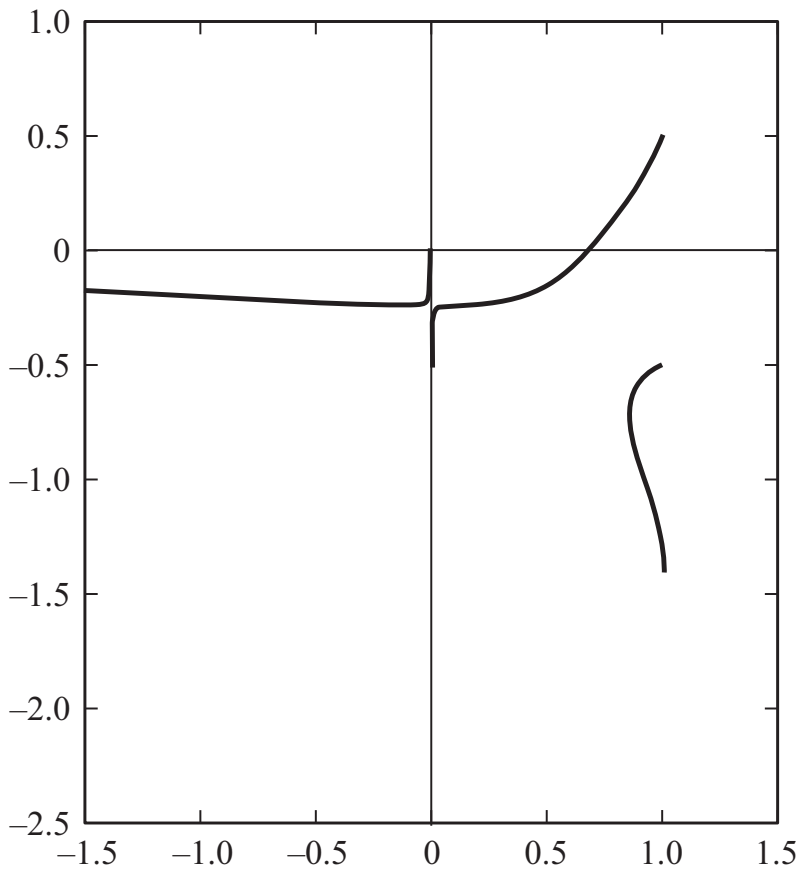

Рис. 2. Траектории корней $\Omega(\tau)$ уравнения (22) при $\beta=1$, $\delta=0.5$ при изменении $\tau$ от нуля до $+\infty$. По осям отложены мнимая (ось ординат) и действительная (ось абсцисс) части $y$.

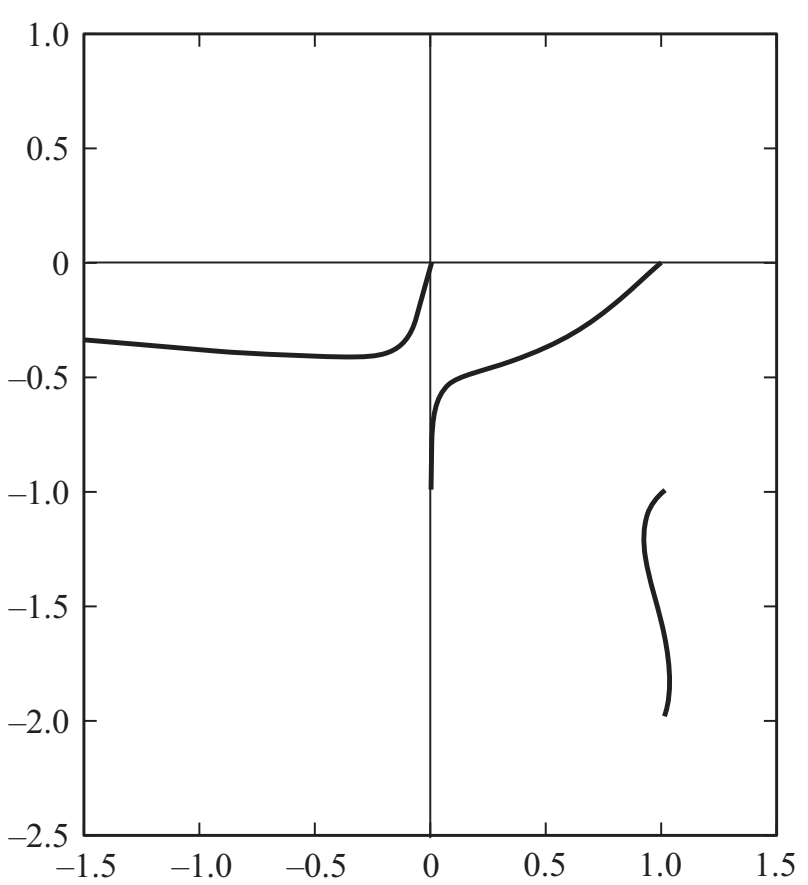

Рис. 3. То же, что и на рис. 2 при $\beta=1, \delta=1$.

Оно сводится к кубическому уравнению для $\Omega$. Решение представлено на рис. 2-4. Видно, что с увеличением параметра $\delta_{i}$, характеризующего степень немонохроматичности ионного пучка при

$$
\delta_{i}>\delta_{C R}
$$




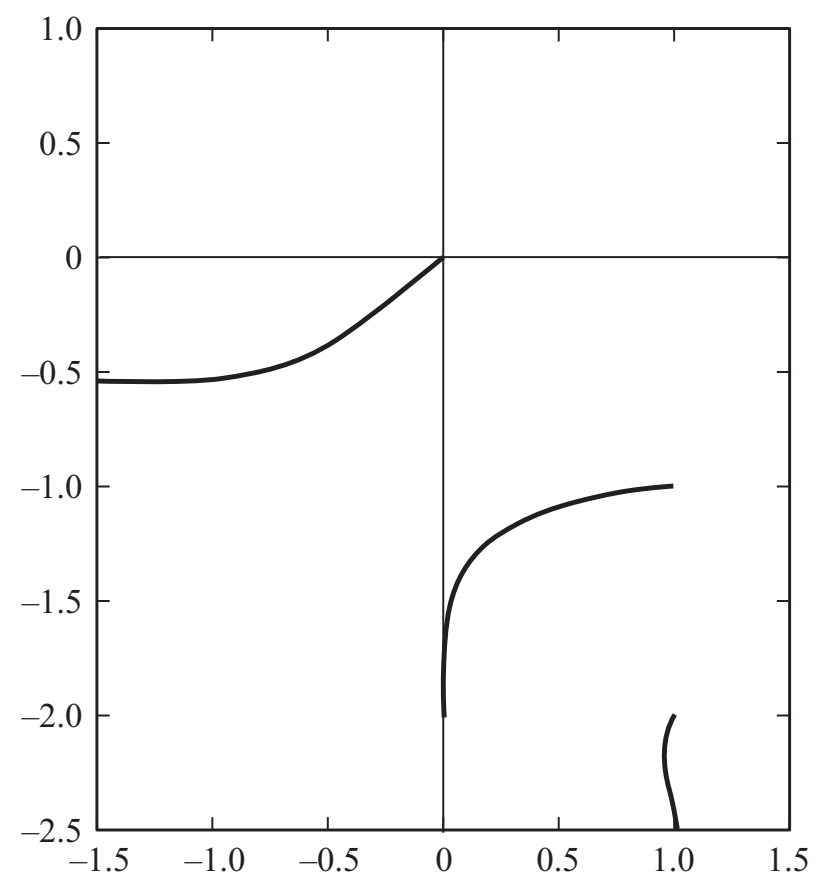

Рис. 4. То же, что и на рис. 2 при $\beta=1, \delta=1$.

в верхней полуплоскости у корни отсутствуют, т.е. наступает устойчивость ионного кольца. Конкретно это происходит, когда уходит из верхней полуплоскости верхний конец средней кривой. Пользуясь подсказкой компьютера (хотя это несложно установить и аналитически), заключаем, что это происходит при $\tau \rightarrow+\infty$. В этом пределе уравнение (22) упрощается и принимает вид

что равносильно

$$
\tau \approx-\frac{1+i \sqrt{\beta}}{2\left[\Omega-1+i\left(\delta_{i}-\sqrt{\beta}\right)\right]},
$$

$$
\Omega \approx 1-i\left(\delta_{i}-\sqrt{\beta}\right)-\frac{p}{2 \tau},
$$

где $p=1+i \sqrt{\beta}$. Из этих уравнений видно, что

$$
\delta_{C R}=\sqrt{\beta}
$$

Магнитное поле, создаваемое кольцом, порядка

$$
B_{R I N G} \sim \frac{2 \pi q n_{i} u r_{B_{i}}}{c}
$$

где $r_{B_{i}}=q B / M c-$ характерный радиус кольца. Отсюда следует оценка

$$
\delta_{i} \sim \beta
$$

Сопоставляя ее с условием (23), заключаем, что АИЦН подавляется при условии, совпадающем с (14), т.е. в кольце с обращенным полем.

\section{4. Учет разброса циклотронных частот для электронов}

Роль этого обстоятельства несложно учесть, приняв для электронов распределение, аналогичное (16).
Тогда слагаемое $\omega_{e}^{2} / \omega_{B_{e}}$ в формуле (18) заменится на $\omega_{e}^{2} /\left(\omega_{B_{e}}-i \Delta \omega_{B_{e}}\right)$, что приводит снова к уравнению (24) с параметром

$$
p=1+\sqrt{\beta} \frac{i-\delta_{e}}{1+\delta_{e}^{2}},
$$

где $\delta_{e}=\Delta \omega_{B_{e}} / \omega_{B_{e}}$. Отсюда ясно, что результаты (23) и (25) не меняются, что является следствием соотношения $\omega_{B_{e}} \gg \omega_{B_{i}}$. Разница лишь в изменении угла, под которым средняя кривая подходит к своему верхнему концу, соответствующему $\tau=+\infty$.

Таким образом, в отличие от рассмотренной выше пучковой неустойчивости, электроны не влияют на характер развития АИЦН, что согласуется с выводами [22].

\section{5. Учет конечности продольного размера $L_{R}$ кольца}

Согласно разд. 2, в интересующем нас случае (1) вследствие обращения направления поля внутри кольца неустойчивость не возникает как при его сжатии, так и при последующем ускорении.

Теперь рассмотрим случай

$$
\beta \lesssim 1
$$

За время порядка периода обращения ионов, $\sim 1 / \omega_{B_{i}}$, АИЦ волна распространяется на расстояние порядка своей длины $\sim \lambda_{A} \sim u_{A} / \omega_{B_{i}}=C / \omega_{i}$. Эта волна не успевает сформироваться, если длина кольца $L_{R}$ в продольном направлении достаточно мала: $L_{R} \lesssim \lambda$. Отсюда можно заключить, что АИЦН не возникает, если $[29,30]$

$$
\frac{L_{R} \omega_{i}}{c} \lesssim 1
$$

Строгий анализ дает [30]

$$
\frac{L_{R} \omega_{i}}{c}<\sqrt{3}
$$

Для начального состояния кольца до его ускорения из этого условия следует ограничение $L_{R}<4 \mathrm{~cm}$, что в проекте [10] выполняется. Критерий Черноштанова-Цыдулько (28) в нашем случае нарушается при увеличении плотности частиц в кольце на порядок, т.е. примерно при трехкратном радиальном его сжатии. Но для достижения энергии ионов $\sim 1 \mathrm{GeV}$, как в проекте [10], кольцо надо радиально сжать в 230 раз, поэтому возникает вопрос об устойчивости дальнейшего сжатия кольца, которое при этом можно считать длинным и тонким цилиндром.

\section{6. Учет экранировки поля АИЦ лайнером}

Учтем влияние токов, наведенных полем возбуждаемой АИЦ волны в плазме лайнера, на устойчивость процесса ускорения кольца. В опыте [5] магнитный поток, 
захваченный лайнером, сохранялся при его сжатии. Это значит, что лайнер можно рассматривать, как идеальный проводник, играющий роль волновода. Это подтверждается и оценками. Так, при трехкратном радиальном сжатии температура электронов составит $\sim 10 \mathrm{eV}$, а толщина скин-слоя на соответствующей такому сжатию циклотронной ионной частоте $\omega_{B_{i}} \sim 3 \cdot 10^{8} \mathrm{~cm}^{-1}$, характерной для альвеновской волны, будет малой по сравнению с толщиной лайнера, $\sim 10^{-3}-10^{-4} \mathrm{~cm}$.

Влияние наведенных в лайнере токов оценим на основе простой модели, в которой поперечное сечение лайнера представляет квадрат со стороной $2 R$, где $R$ примерно равен радиусу лайнера.

Сначала будем считать, что $r_{B_{i}} \ll R$, и что сжимаемая плазма вплотную прилегает к стенкам лайнера. Такая модель позволяет провести аналитический расчет. Полученные в этом приближении результаты позволят получить количественные и качественные оценки и для интересующего нас случая $r_{B_{i}} \approx R$.

Начнем со случая холодной плазмы

$$
k r_{B_{i}} \ll 1
$$

(для электронов в интересующем нас случае подобное соотношение, $k r_{B_{i}} \ll 1$, выполнено всегда). Из уравнений Максвелла для полей $\mathbf{E}$ и $\mathbf{B}$ в возбуждаемой АИЦ волне получаем

$$
\begin{gathered}
(\nabla \times \mathbf{B})_{\alpha}=-i \frac{\omega}{c} \varepsilon_{\alpha \beta} E_{\beta}, \\
(\nabla \times \mathbf{E})=i \frac{\omega}{c} \mathbf{B},
\end{gathered}
$$

где $\alpha, \beta=x, y$, по повторяющимся индексам идет суммирование, а начало координат $(x, y)$ располагаем в центре поперечного сечения лайнера. Здесь учтено, что в интересующем нас случае $\omega \sim \omega_{B_{i}} \ll \omega_{e}$ продольное (вдоль основного магнитного поля) электрическое поле в кольце экранируется электронами и поэтому его можно считать равным нулю. При $\mathbf{k} \| \mathbf{B}$ это же верно и для В. Нужные нам компоненты тензора диэлектрической проницаемости плазмы в случае (29) равны [31]

$$
\begin{gathered}
\varepsilon_{x x}=\varepsilon_{y y} \equiv \varepsilon=1-\frac{\omega_{e}^{2}}{\omega^{2}-\omega_{B_{e}}^{2}}-\frac{\omega_{i}^{2}}{\omega^{2}-\omega_{B_{i}}^{2}} \\
\approx-\frac{\omega_{e}^{2}}{\omega^{2}}-\frac{\omega_{i}^{2}}{\omega^{2}-\omega_{B_{i}}^{2}}, \\
\varepsilon_{x y}=-\varepsilon_{y x}=i g, \\
=\frac{\omega_{B_{e}} \omega_{e}^{2}}{\omega\left(\omega^{2}-\omega_{B_{e}}^{2}\right)}-\frac{\omega_{B_{i}} \omega_{i}^{2}}{\omega\left(\omega^{2}-\omega_{B_{i}}^{2}\right)} \\
\approx-\frac{\omega_{e}^{2}}{\omega \omega_{B_{e}}}-\frac{\omega_{B_{i}} \omega_{i}^{2}}{\omega\left(\omega^{2}-\omega_{B_{i}}^{2}\right)} .
\end{gathered}
$$

Исключая из (30) и (31) магнитное поле, с учетом зависимостей $(\mathbf{E}, \mathbf{B}) \propto \exp (i k z-i \omega t)$, получаем следующую систему уравнений для компонент электрического поля в АИЦ

$$
\left\{\begin{array}{l}
\Delta_{\perp} E_{x}+\left(\frac{\omega^{2}}{c^{2}} \varepsilon-k^{2}\right) E_{x}+i g E_{y}=0, \\
\Delta_{\perp} E_{y}+\left(\frac{\omega^{2}}{c^{2}} \varepsilon-k^{2}\right) E_{y}+i g E_{x}=0 .
\end{array}\right.
$$

где

$$
\Delta_{\perp}=\frac{\partial^{2}}{\partial x^{2}}+\frac{\partial^{2}}{\partial y^{2}} .
$$

Собственные моды волновода, образуемого лайнером, ищем в виде

$$
\left\{\begin{array}{l}
E_{x}=C_{1} \cos \left(\kappa_{x} x\right) \cos \left(\kappa_{y} y\right), \\
E_{y}=C_{2} \cos \left(\kappa_{x} x\right) \cos \left(\kappa_{y} y\right) .
\end{array}\right.
$$

Электрическое поле на поверхностях лайнера должно быть направлено перпендикулярно к ним, поэтому

$$
\kappa_{x}=\frac{\pi}{R}\left(\frac{1}{2}+n_{x}\right), \quad \kappa_{y}=\frac{\pi}{R}\left(\frac{1}{2}+n_{y}\right),
$$

где

$$
n_{x}, n_{y}=0,1,2, \ldots
$$

В действительности нас интересует случай $r_{B_{i}} \approx R$, когда размер поперечных неоднородностей полей $\mathbf{E}$ и $\mathbf{B}$ в возбуждаемой ионным кольцом АИЦ волне порядк $R$, чему соответствует только основная мода $n_{x}=n_{y}=0$. Поэтому

$$
\begin{gathered}
\kappa_{x}=\kappa_{y}=\frac{\pi}{2 R}, \\
\kappa^{2}=\kappa_{x}^{2}+\kappa_{y}^{2}=\frac{\pi^{2}}{2 R^{2}} .
\end{gathered}
$$

Из (32) и (33) следует дисперсионное уравнение для движущейся внутри лайнера АИЦ

$$
\frac{q^{2} c^{2}}{\omega^{2}}=\varepsilon+g \approx-\frac{1}{\omega}\left(\frac{\omega_{e}^{2}}{\omega_{B_{e}}}+\frac{\omega_{i}^{2}}{\omega-\omega_{B_{i}}}\right),
$$

где

$$
q^{2}=\kappa^{2}+k^{2}=\frac{\pi^{2}}{2 R^{2}}+k^{2} .
$$

Согласно разд. 2, с учетом немонохроматичности ионов и при конечном значении параметра $k r_{B_{i}}$ уравнение (34) следует переписать в виде

$$
\frac{q^{2} c^{2}}{\omega^{2}} \approx-\frac{F(\omega)}{\omega}
$$

где функция $F(\omega)$ определена формулами (19), (20). Это уравнение приводится к виду (22) после перехода к безразмерным величинам (7), но теперь

$$
\tau=\frac{q^{2} c^{2}}{\omega_{i}^{2}} .
$$

Как было показано в разд. 3 , при условии $\delta_{i}>\sqrt{\beta}$, которое в нашем случае сводится к (14), в верхней 


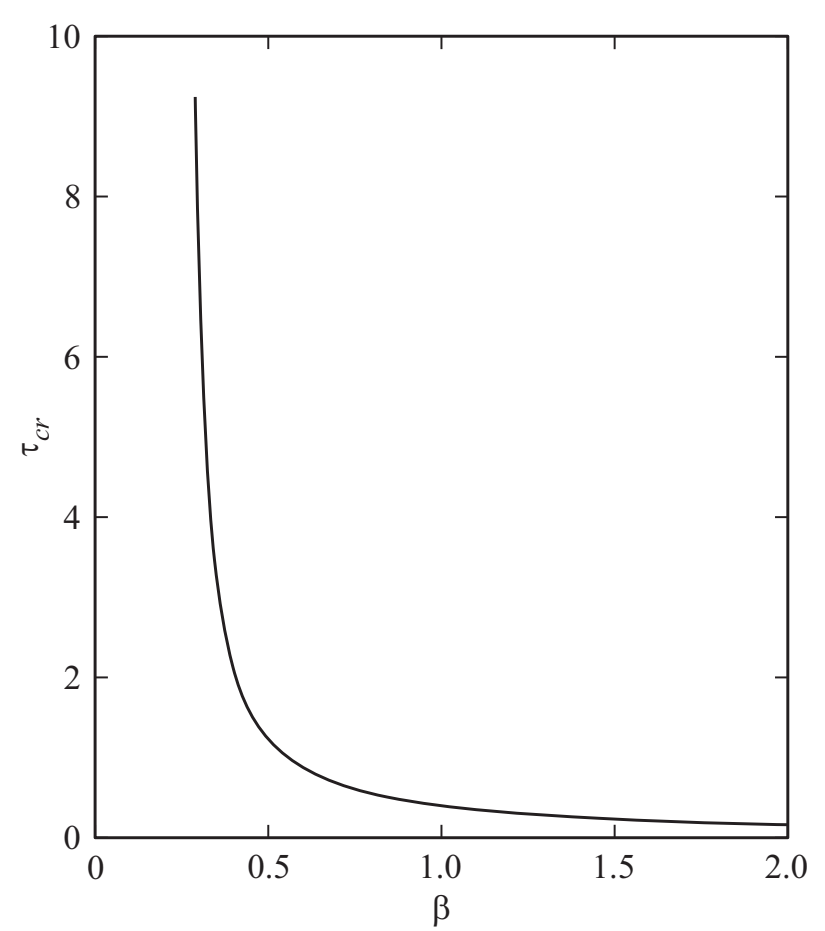

Рис. 5. Величина параметра $\tau_{C R}$ в зависимости от $\beta$ при $\delta_{i}=0.5$.

полуплоскости комплексной переменно́й $\Omega$ при всех значениях параметра отсутствуют корни уравнения (36), т. е. ускорение устойчиво.

Теперь рассмотрим случай

$$
\delta_{i}<\sqrt{\beta}
$$

который, согласно (26), равносилен

$$
\beta<1
$$

Перепишем уравнение (22) в виде

$$
\left(\Omega-1+i \delta_{i}\right)^{2}+\frac{\Omega\left(\Omega+i \delta_{i}\right)}{\tau}\left(\Omega-1+i \delta_{i}\right)+\beta=0 .
$$

Устойчивость имеется, когда

$$
\Omega^{\prime \prime}<0
$$

т. е. при условии

$$
\tau<\tau_{C R}
$$

Зависимость параметра $\tau_{C R}$ от $\beta$ в случае $\delta_{i}=0.5$ приведена на рис. 5.

С целью получить условие устойчивости в аналитическом виде предположим заранее (ниже это подтвердится), что в случае (38) устойчивость ускорения ионного кольца за счет экранировки АИЦ лайнером если и возможна, то только при

$$
\tau \gg 1
$$

В нулевом приближении по малому параметру $1 / \tau$ из (40) получаем

$$
\Omega \approx 1-i\left(\delta_{i}-\sqrt{\beta}\right),
$$

причем здесь взят корень, определяющий наличие или отсутствие устойчивости (он соответствует средней кривой на рис. 2-4). В следующем приближении по $1 / \tau$ получаем

$$
\Omega \approx \Omega^{\prime}+i \Omega^{\prime \prime}
$$

где

$$
\begin{gathered}
\Omega^{\prime}=1-\frac{1+\beta-\delta_{i} \sqrt{\beta}}{2 \tau}, \\
\Omega^{\prime \prime}=-\delta_{i}+\sqrt{\beta}-\frac{2 \sqrt{\beta}-\delta_{i}}{2 \tau} .
\end{gathered}
$$

Отсюда следует, что

$$
\tau_{C R}=\frac{2 \sqrt{\beta}-\delta_{i}}{2\left(\sqrt{\beta}-\delta_{i}\right)} \approx \frac{\sqrt{\beta}}{2\left(\sqrt{\beta}-\delta_{i}\right)} .
$$

Эта формула верна, когда $\tau_{C R} \gg 1$, т. е. при

$$
1-\frac{\delta_{i}}{\sqrt{\beta}} \ll 1
$$

Согласно (13), $k \lesssim 1 / r_{B_{i}} \sim 1 / R$, поэтому

$$
q^{2}=\frac{\pi^{2}}{2 R^{2}}+k^{2} \lesssim \frac{5}{R^{2}} .
$$

Вместе с (37) и (41) это приводит к следующему условию подавления АИЦН токами, наведенными в лайнере в кольцах из семейства (39)

$$
R \gtrsim \frac{3 c}{\omega_{i}} \sqrt{1-\frac{\delta_{i}}{\sqrt{\beta}}}
$$

Как говорилось выше, если это условие выполнялось вначале, то оно будет выполнено и в процессе ускорения кольца, поскольку параметры $R \omega_{i} / c, \delta_{i}$ и $\beta$ остаются при этом постоянными. Отсюда заключаем, что экранировка лайнером будет подавлять АИЦН в течение всего процесса, если в начальная геометрия ускорителя такова

$$
\left(\frac{R}{L_{R}}\right)_{\text {initial }} \gtrsim \sqrt{3\left(1-\frac{\delta_{i}}{\sqrt{\beta}}\right)}
$$

Видно, что в случае (38) энергетический разброс пучка ионов должен быть не слишком малым - так, при разумном требовании

$$
\left(\frac{R}{L_{R}}\right)_{\text {initial }} \lesssim 1
$$

должно быть

$$
1-\frac{\delta_{i}}{\sqrt{\beta}} \lesssim \frac{1}{3}
$$


В схеме ускорителя $[1,2]$ подразумевается условие

$$
\frac{R}{r_{B_{i}}} \sim 1-2
$$

оказывающееся гораздо более жестким, чем геометрическое требование (46). Действительно, из (45) следует соотношение

$$
\frac{R}{r_{B_{i}}} \sim \frac{3 \omega_{B_{i}} c}{\omega_{i} u} \sqrt{1-\frac{\delta_{i}}{\sqrt{\beta}}}
$$

которое в случае проекта [10] имеет вид

$$
\frac{R}{r_{B_{i}}} \sim 300 \sqrt{1-\frac{\delta_{i}}{\sqrt{\beta}}} .
$$

Ясно, что его трудно совместить с (47).

\section{Выводы}

Суммируя полученные результаты, приходим к следующим выводам:

1) ускорение ионных колец сжимающимися лайнерами устойчиво только при $\beta \sim 1$ (см. определение $(1))$, когда кольцо представляет собой конфигурацию с обращенным магнитным полем (a field-reversed configuration - FRC);

2) длина лайнера $L_{R}$ должна удовлетворять критерию Черноштанова-Цыдулько (28);

3) токи, индуцируемые в лайнере возбуждаемой ионами альвеновской ионно-циклотронной волной, оказывают стабилизирующее воздействие на рост ее амплитуды.

\section{Конфликт интересов}

Авторы заявляют, что у них нет конфликта интересов.

\section{Список литературы}

[1] Меньшиков Л.И., Недосеев С.Л., Смирнов В.П., Сомов Л.Н. О возможности получения высокоэнергичных электронных и ионных колец в сжимающихся лайнерах. Препринт ИАЭ-5077/6. М. 1990.

[2] Меньшиков Л.И., Недосеев С.Л., Смирнов В.П., Сомов Л.Н. // АЭ. 1991. Т. 71. № 6. С. 511.

[3] Dreike P.L., Greenly J.B., Hammer D.A., Sudan R.N. // 1981. Vol. 46. N 8. P. 539.

[4] Dreike P.L., Greenly J.B., Hammer D.A., Sudan R.N. // Phys. Fluid. 1982. Vol. 25. N 1. P. 59.

[5] Felber F.S., Malley M.M., Wessel F.J., Matzen M.K., Palmer M.A., Spielman R.B., Liberman M.A., Velikovich A.L. // Phys. Fluid. 1988. Vol. 31. N 7. P. 2053.

[6] Петров Н.Н. // Атом. 2008. Т. 37. № 2.

[7] Alekseev P.N., Ignatiev V.V., Konakov S.A., Menshikov L.I., Ponomarev-Stepnoi N.N., Prusakov V.N., Stukalov V.A., Subbotine S.A. // Nucl. Eng. Design. 1997. Vol. 173. N 1-3. P. 151.
[8] Микаэлян Л.А., Спивак П.Е., Циноев В.Г. // ЯФ. 1965. Т. 1. № 5. C. 853 .

[9] Ляшук В.И., Лютостанский Ю.С. // Письма в ЖЭТФ. 2016. T. 103. № 5. С. 331.

[10] Bystritskii V., Wessel F.J., Rostoker N., Rahman H. Novel staged $z$-pinch concept as super radiant $X$-ray source for ICF. In: Current Trends in International Fusion Research, Springer. 1997. P. 347-364.

[11] Wessel F.J., Coleman P.L., Loter N., Ney P., Rahman H.U., Rauch J., Thompson J. // J. Appl. Phys. 1997. Vol. 81. P. 340.

[12] Дзарахохова А.С., Зареикий Н.П., Максимычев А.В., Меньшиков Л.И., Меньшиков П.Л. // ЖЭТФ. 2020. Т. 157. № 1. C. 165.

[13] Baldwin D.E., Rensink M.E. // Comments on Plasma Phys. Controlled Fusion. 1978. Vol. 4. P. 55.

[14] Sudan R.N. // AIP Conf. Proceed. 1994. Vol. 311. N 1. P. 194.

[15] Tuszewski M. // Nucl. Fusion. 1988. Vol. 28. N 11. P. 2033.

[16] Steinhauer L.C. // Phys. Plasm. 2011. Vol. 18. P. 070501.

[17] Wang M.Y., Miley G.H. // Nucl. Fusion. 1979. Vol. 19. N 1. P. 39.

[18] Larrabee D.A., Lovelace R.V. // Phys. Fluid. 1982. Vol. 25. N 4. P. 714

[19] Steinhauer L.C. // Phys. Plasma. 2011. Vol. 18. N 7. P. 070501.

[20] Lovelace R.V. // Phys. Fluid. 1979. Vol. 22. N 3. P. 542.

[21] Sudan R.N., Ott E. // Phys. Rev. Lett. 1974. Vol. 33. N 6. P. 355.

[22] Davidson R.C., Ogden J.M. // Phys. Fluid. 1975. Vol. 18. N 8. P. 1045.

[23] Tajima T., Mima K., Dawson J.M. // Phys. Rev. Lett. 1977. Vol. 39. N 4. P. 201.

[24] Coensgen F.H., Anderson C.A., Casper T.A. et. al. // Phys. Rev. Lett. 1980. Vol. 44. P. 1132.

[25] Smith G.R. // Phys. Fluid. 1984. Vol. 27. N 6. P. 1499.

[26] Smith G.R. ibid. 1984. Vol. 27. N 8. P. 2120.

[27] Сагдеев Р.З., Шафранов В.Д. // ЖЭТФ. 1960. Т. 39. С. 181.

[28] Weibel E.S. // Phys. Rev. Lett. 1959. Vol. 2. P. 83.

[29] Tajima T., Mima K. // Phys. Fluid. 1980. Vol. 23. N 3. P. 577.

[30] Chernoshtanov I.S., Tsidulko Yu.A. // Fusion Sci. Tech. 2011. Vol. 59. N 1T. P. 116.

[31] Шабранов В.Д. Электромагнитные волны в плазме. В сб. Вопросы теории плазмы. Вып. 3. М.: Госатомиздат, 1963. 